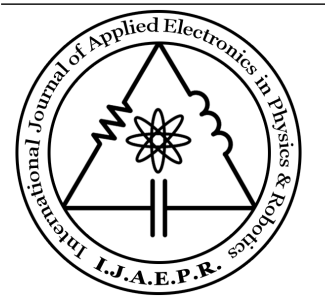

Research Article

\title{
Retarding Forces Cancellation in Electrolyte Solutions-An Electrical Approach
}

\author{
Kaveh Mazloomi ${ }^{1, *}$, Nasri Sulaiman ${ }^{1}$ \\ (1) Department of Electrical and Electronics Engineering, University Putra Malaysia, 43400 Serdang, Selangor, Malaysia.
}

\section{Article history:}

Received 13 May 2013

Reviewed 16 May 2013

Revised 16 May 2013

Accepted 19 May 2013

Published 2 July 2013

\begin{abstract}
The process efficiency of electrolytic hydrogen production is enhanced regarding the transient response of a water electrolysis cell at the time of voltage application and disconnection. When such cells are connected to a power source, a momentary current surge passes through the cell charging the electrical double layer. In addition, the electrolysis process does not stop immediately after the cell is disconnected from its power source. An experimental electrolysis cell was constantly subjected to these phenomena under high frequency pulsating voltage conditions. Results show that the process efficiency is higher when the power is applied in the form of high frequency short pulses. Applied pulse width and frequency were as short as 25 nanoseconds and up to over $7 \mathrm{MHz}$ respectively.
\end{abstract}

Keywords: water electrolysis, efficiency, short pulses, frequency, hydrogen, electrochemical conductance.

\section{Introduction}

Water electrolysis is known as one of the many approaches for hydrogen production. For this electricity dependent process, decreasing power losses has been an important research objective for a long time ${ }^{[1,2]}$. Such objective has been pursued by different approaches mainly, chemical, electrochemical and mechanical. A wide range of electrode and separator materials have been extensively experimented with ${ }^{[3,4]}$ as well as different electrolyte solutions ${ }^{[5,6]}$. Significant literature is available covering subjects like the alignments of electrodes in an electrolytic bath ${ }^{[7]}$, their optimum distance, their surface texture and reaction and methods of forced bubble-plate detachment ${ }^{[8]}$. Although an electrical equivalent circuit for an electrolysis cell is available, records about the possibility of influencing the hydrogen production process efficiency solely regarding the form of power application are noticeably rare to find ${ }^{[5,6,9]}$.

Electrolytes and ionic solutions are second class conductors ${ }^{[10]}$. Therefore, electric current is resulted by the physical movement of charged species (ions) through the bulk of material to the surface of an electrode. Such conductors behave less resistive and more conductive if ions can travel at higher velocities ${ }^{[11]}$. As it is depicted in Fig. 1, ions of an electrolyte have a random, Brownian in nature movement at the absence of an external electric field.

These movements average out in time ${ }^{[10]}$. However, when electrode plates are being charged by an external

\footnotetext{
${ }^{*}$ Corresponding author: K. Mazloomi

厄: +6 0172429060

凶: kavehoo@yahoo.com
}

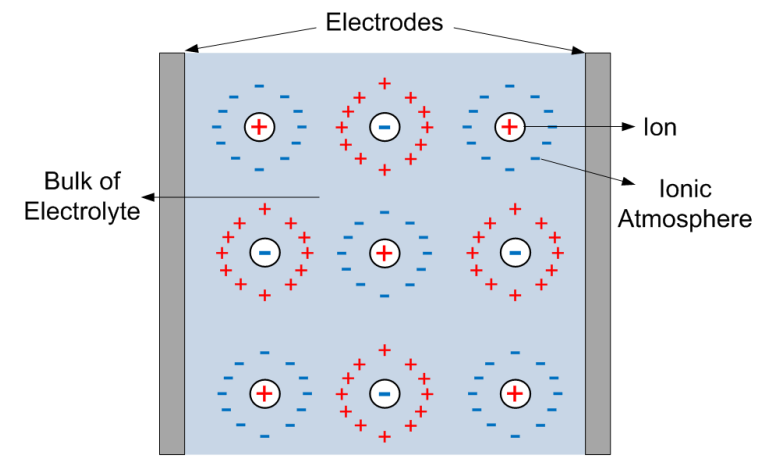

Fig. 1. Ions distribution before voltage application

voltage source, ionic particles of the electrolyte start to move toward their opposite sign charged plate.

This movement is usually slowed down because of retarding forces such as the formation of diffusion and electrical double layers (EDL) ${ }^{[12]}$ and relaxation time ${ }^{[11]}$ and electrophoretic effects ${ }^{[10]}$. The goal of our research was to implement a method of power application to reduce the retarding effects and reduce the resistivity of the electrolyte solution. Therefore, less energy has to be consumed to generate a given mass of hydrogen.

Fig. 2 shows the structure of an electrical double layer and diffusion layer. As in this figure, negative ions are covering the surface of a positively charged electrode plate. The interface between different signed species is called the EDL. On the other hand, the formation of a stable EDL on an electrode plate comes with unwanted side effects. Concentration of in this case negative ions on the surface of the anode attracts positively charged species. Although the charge of one ion is very small, the 


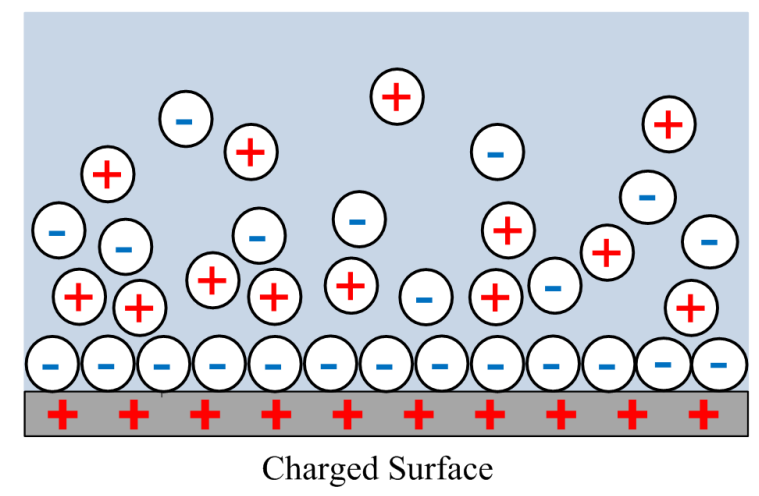

Fig. 2. Ions concentration at the vicinity of an electrode short-range force between two opposite charged particles is significant. The latter is due to the small distance between those particles. These forces cause an accumulation of unwanted charges at the vicinity of an electrode (in this case, positive ions near positive plate). Moreover, when the concentration of a desired species is increased near the surface of an electrode plate, the accumulated charges repel similar particles and force them back to the bulk of electrolyte. Area where species are present at concentrations higher than the bulk of electrolyte is called the diffusion layer. A diffusion layer mainly forms by short range forces between charged species as explained. Shimizu et al. ${ }^{[12]}$ calculated a steady EDL is formed not earlier than tens of milliseconds after an electric field is applied to an electrolytic bath. Furthermore, a time span of a few microseconds is required for a diffusion layer to be formed.

As it is shown in Fig. 3, an ion travels toward its regarding electrode plate under the influence of an electric field. However, when its journey starts, the ionic atmosphere surrounding a central ion does not follow its motions instantaneously. The delay between the movement of a central ion and the formation of a symmetrical ionic atmosphere around it is called the time of relaxation. When an ion is surrounded by an asymmetrical ionic atmosphere, its opposite sign charged ionic atmosphere apply a retarding force against its velocity axis. Therefore, a fraction of the external energy provided to the system between the starting movement phase of the central ion and the complete formation of its ionic atmosphere is consumed to overcome this force. According to Koryta et al. ${ }^{[10]}$ the time of relaxation is a matter of several hundred nanoseconds depending on the electrolyte dilution and strength of the applied external field.

In addition to the above-mentioned, an ionic atmosphere itself is subjected to attraction forces from the opposite charged electrode. This phenomenon causes further velocity reduction in the process of ionic movement.

\section{Methodology}

The idea behind the experimental work of this research was to apply voltage pulses short enough to prevent the formation of steady layers. Moreover, short repetitive pulse application makes us able to stop the energy flow to the system during the relaxation time periods. For the mentioned reasons, an electrolysis cell was sat up as is shown in Fig. 4.

The cell consists of a single compartment container filled with potassium hydroxide in distilled water. A pair of $20 \times 40 \times 0.5 \mathrm{~mm}$ aluminum plates were utilized to serve as electrodes. The output hydroxygen contains some unwanted moisture. This gas mixture is guided through a spiral shaped copper tubing almost 2.5 meters long, surrounded with by to condense and become dryer. This action is mainly a protective precaution for downstream sensors which are designed to perform readings on dry gasses. The gas mixture is fed to a container equipped with a temperature and a relative humidity $(\mathrm{RH})$ sensor. According to the definition of $\mathrm{RH}$ (1), the partial pressure of water vapor and as a result, the humidity content can be calculated.

$$
\phi \%=\frac{p_{w}}{P_{w s}} \times 100
$$

where $P_{w}$ is the vapor partial pressure, $P_{w s}$ is the saturation vapor partial pressure and $\phi$ is the relative humidity percentage. A digital gas flow meter is placed after the first sensor container with an absolute pressure meter connected between these compartments. Data acquired from all sensors is fed to analog to digital inputs and digital communication ports of a microcontroller. The collected instrumentation readings are displayed on an LCD unit where they can also be uploaded to a computer. For each experimental procedure, the data are substituted in the ideal gas equation as below

$$
P V=n R T
$$

where $T$ is the temperature in Kelvins, $R$ is the universal gas constant is equal to $0.0820577 \mathrm{~L}$ atm mol ${ }^{-1} \mathrm{~K}^{-1}$, $n$ is the number of the moles of gas, $V$ is its volume in liters and $\mathrm{P}$ is its pressure in atmospheres. Knowing the moisture content, and the fact that according to reaction equation $\left(\mathrm{H}_{2} \mathrm{O} \rightarrow \mathrm{H}_{2}+\frac{1}{2} \mathrm{O}_{2}\right), \frac{2}{3}$ of gas production is hydrogen; it is easy to calculate the number of hydrogen moles per time unit. Since the mass of each mole of hydrogen is $2.0158 \mathrm{~g}$, and each kilogram of this substance contains an energy equivalent of $39.39 \mathrm{kWh}$, we can calculate a 2363.4 Watt minutes of energy being contained in a gram of hydrogen. Equation (3) is used to calculate the mass of hydrogen production.

$$
m=\frac{P \times\left(P P_{H O}\right) \times \frac{2}{3} \times \frac{1}{1000}}{0.0820577 \times T} \times 2.0158
$$

where $P P_{H O}$ is the partial pressure of hydroxygen gas mixture, $T$ is Temperature in Kelvins, $P$ is the pressure reading of the sensor and $m$ is the mass of hydrogen being produced. The nominator of the fraction is divided by a factor of 1000 since the flow sensor reports a value with

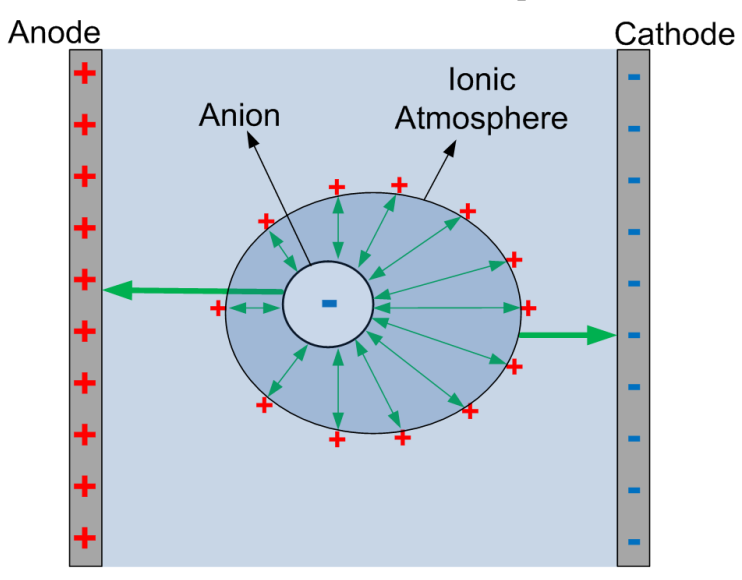

Fig. 3. Relaxation time and electrophoretic effects 


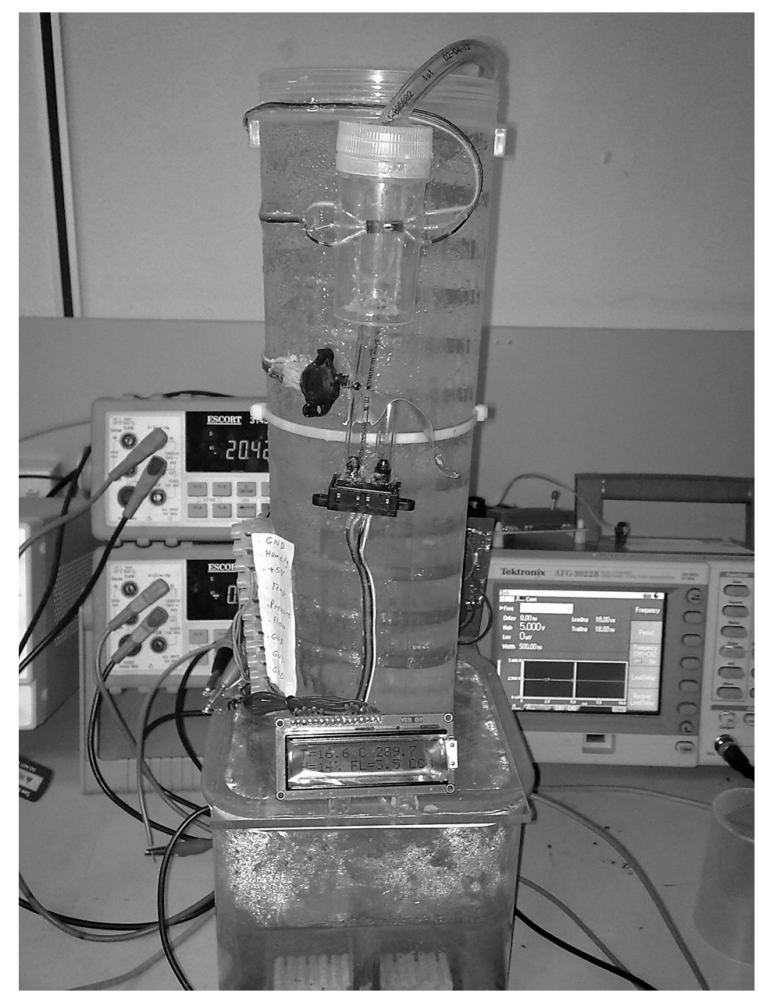

Fig. 4. Experimental cell setup

a dimension of cubic centimeters per minute (CCM) and the dimension of universal gas constant is expressed by liters as the volumetric term.

Knowing the hydrogen production rate, (4) was introduced to measure the efficiency percentage of the process as below

$$
\eta \%=\frac{E_{H_{2}}(t)}{E_{s}(t)} \times 100
$$

Where $\eta$ is the efficiency in percent, $E_{s}$ is the energy drawn from the power supply during a certain time span and $E_{\mathrm{H}_{2}}$ is the gravimetric energy content of the produced hydrogen during the same period. The experiments were repeated on the same electrolysis cell setup at different input voltages ranging between 2 and 10 Volts and within a wide frequency range. The experimental results are demonstrated in the following section.

\section{Results and Discussions}

Fig. 5 depicts the process efficiency for different input voltages and at various test duty cycles. Duty cycle states the proportion of the time power is being applied to the cell to total overall signal period at a certain frequency.

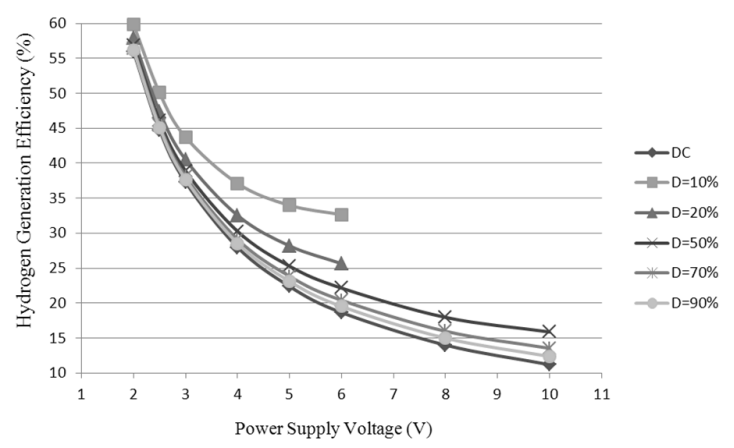

Fig. 5. Duty cycle and voltage dependence of production efficiency behavior at different input voltages

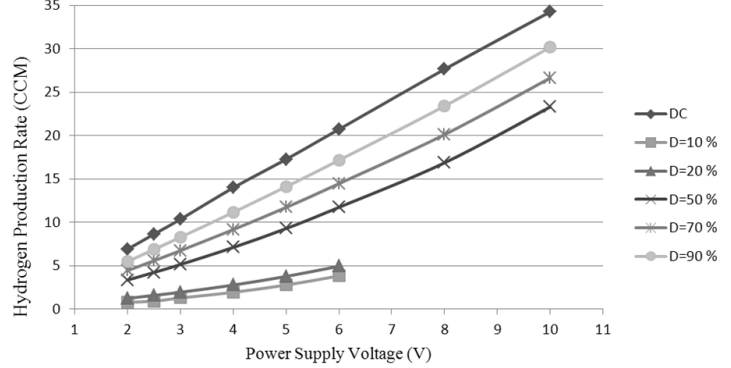

Fig. 6. Duty cycle and voltage dependence of production efficiency behavior at different input voltages

As it is illustrated in this figure, the process of electrolytic hydrogen generation takes place more efficiently at lower voltages and reduced duty cycles. As it was discussed earlier, when the period of voltage application is shortened enough, there will be not enough time for a stable electrical double layer and diffusion layer to form. Moreover, the electrolysis process cannot be started unless the applied voltage is larger than the reversible voltage ${ }^{[13]}$. As it was mentioned earlier, the ideal minimum theoretical required voltage for water electrolysis is approximately $1.23 \mathrm{~V}$. However, the practical minimum required voltage for electrolytic hydrogen production is higher than $1.48 \mathrm{~V}^{[14,15]}$. The experimental electrolysis cells of this work were unable to produce a measurable volume of gas unless the supply voltage was set to a value larger than $1.77 \mathrm{~V}$.

In addition, Fig. 6 illustrates the hydrogen production rates for the same test conditions as discussed earlier. As it is shown in Fig. 5 and 6 , to increase the production of the process of electrolytic hydrogen generation, its efficiency has to be sacrificed. The latter is since the amount of energy provided to an electrochemical cell per unit time is remarkably decreased while the power is being applied in the form of short pulses. The shorter the pulses get, the more time is required to produce the same mass of hydrogen as in DC or higher duty cycle modes. However there is an optimum efficiency production rate for each condition. This is the intersection point of the production rate and efficiency graphs.

\section{Conclusion}

As a result of the experimental work, it can be expressed that applying short pulses to a water electrolysis cell instead of a constant DC signal will lead to a more efficient water decomposition process. According to available literature, the ideal short pulse duration is calculated to be not longer than several nanoseconds and it depends on the built of the electrochemical cell. The frequency of pulse application also has an influence on the process efficiency. This is caused by the interaction between the externally applied field and the field strength of the ionic atmosphere of individual electrolyte particles and the interaction between electrolyte species themselves. On the other hand, the enhanced efficiency comes along with lower production rate since less energy is provided to the cell in such cases. This problem can be solved by using a number of lower capacity electrolysis cells instead of one ${ }^{[16,17]}$.

\section{REFERENCES}

[1] J. W. Richards, "Modern theories of electrolysis," Journal of the Franklin Institute, vol. 141, no. 3, pp. 192-218, 1896. 
[2] - "Secondary reactions in electrolysis," Journal of the Franklin Institute, vol. 152, no. 3, pp. 201-224, 1901.

[3] R. Renaud and R. L. LeRoy, "Separator materials for use in alkaline water electrolysers," International Journal of $\mathrm{Hy}$ drogen Energy, vol. 7, no. 2, pp. 155-166, 1982.

[4] R. P. Viswanath, "A patent for generation of electrolytic hydrogen by a cost effective and cheaper route," International journal of hydrogen energy, vol. 29, no. 11, pp. 1191-1194, 2004.

[5] S. K. Mazloomi and N. Sulaiman, "Influencing factors of water electrolysis electrical efficiency," Renewable and Sustainable Energy Reviews, vol. 16, no. 6, pp. 4257-4263, 2012.

[6] K. Mazloomi, N. B. Sulaiman, and H. Moayedi, "Electrical efficiency of electrolytic hydrogen production," International Journal of Electrochemical Science, vol. 7, pp. 33143326, 2012.

[7] N. Nagai, M. Takeuchi, T. Kimura, and T. Oka, "Existence of optimum space between electrodes on hydrogen production by water electrolysis," International journal of hydrogen energy, vol. 28, no. 1, pp. 35-41, 2003.

[8] S. D. Li, C. C. Wang, and C. Y. Chen, "Water electrolysis in the presence of an ultrasonic field," Electrochimica Acta, vol. 54, no. 15, pp. 3877-3883, 2009.

[9] A. Ursúa, L. Marroyo, E. Gubía, L. M. Gandía, P. M. Diéguez, and P. Sanchis, "Influence of the power supply on the energy efficiency of an alkaline water electrolyser," international journal of hydrogen energy, vol. 34 , no. 8, pp. 3221-3233, 2009.
[10] J. Koryta, J. Dvorak, and L. Kavan, Principles of electrochemistry. Wiley Chichester (W. Sx) etc., 1993.

[11] V. S. Bagotsky, Fundamentals of electrochemistry. WileyInterscience, 2005, vol. 44.

[12] N. Shimizu, S. Hotta, T. Sekiya, and O. Oda, "A novel method of hydrogen generation by water electrolysis using an ultra-short-pulse power supply," Journal of applied electrochemistry, vol. 36, no. 4, pp. 419-423, 2006.

[13] Ø. Ulleberg, "Modeling of advanced alkaline electrolyzers: a system simulation approach," International Journal of Hydrogen Energy, vol. 28, no. 1, pp. 21-33, 2003.

[14] M. K. Hansen, D. Aili, E. Christensen, C. Pan, S. Eriksen, J. O. Jensen, J. H. von Barner, Q. Li, and N. J. Bjerrum, "Pem steam electrolysis at $130^{\circ} \mathrm{c}$ using a phosphoric acid doped short side chain pfsa membrane," International Journal of Hydrogen Energy, 2012.

[15] S. Siracusano, V. Baglio, N. Briguglio, G. Brunaccini, A. Di Blasi, A. Stassi, R. Ornelas, E. Trifoni, V. Antonucci, and A. S. Aricò, "An electrochemical study of a pem stack for water electrolysis," International Journal of Hydrogen Energy, vol. 37, no. 2, pp. 1939-1946, 2012.

[16] K. Mazloomi and C. Gomes, "Hydrogen as an energy carrier: prospects and challenges," Renewable and Sustainable Energy Reviews, vol. 16, no. 5, pp. 3024-3033, 2012.

[17] K. Mazloomi and N. B. Sulaiman, "Efficiency enhancement of pwm controlled water electrolysis cells," World Academy of Science, Engineering and Technology, vol. 74, pp. 634638, 2011. 